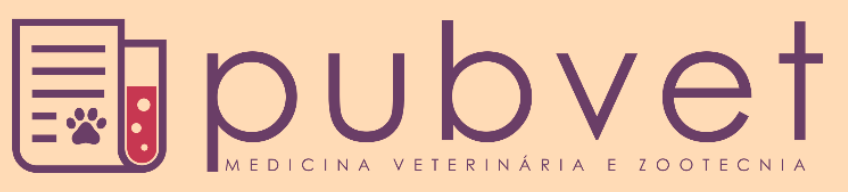

ISSN $1982-1263$

https://doi.org/10.31533/pubvet.v14n1a499.1-4

\title{
Hérnia perineal bilateral em uma gata: relato de caso
}

\author{
Selmar Moreira ${ }^{1}$, Francisco Lima Silva ${ }^{2 *}{ }^{\bullet}$, Catarina Rafaela Alves da Silva ${ }^{3}$, Aline Simão Holanda \\ Conceição Alves e Ferreira ${ }^{4} \bullet$, Laide Danielle Coelho da Silva Chaves $^{4}{ }^{\bullet}$, Laíz Pinheiro Santos ${ }^{5} \bullet$ \\ ${ }^{1}$ Médico Veterinário, Clínica Veterinária Animal's, Teresina-PI Brasil. \\ ${ }^{2}$ Professor da Universidade Federal do Piauí, Departamento de Clínica e Cirurgia Veterinária. Teresina-PI Brasil. \\ ${ }^{3}$ Médica Veterinária Anestesiologista/Intensivista do Hospital Veterinário Universitário da Universidade Federal do Piauí, Teresina-PI Brasil. \\ ${ }^{4}$ Discente do curso de Medicina Veterinária em Universidade Federal do Piauí. Teresina-PI Brasil. \\ ${ }^{5}$ Graduanda em Medicina Veterinária, Universidade Federal do Piauí, Teresina-PI Brasil. \\ *Autor para correspondência, E-mail: flimavet@hotmail.com
}

Resumo. Hérnia perineal é descrita como o enfraquecimento muscular da região do períneo, podendo assim ocorrer a migração de vísceras para a região. Ocorre principalmente em cães, machos, não castrados e idosos. É pouco frequente em felinos. Um felino, fêmea, SRD, 7 anos, foi atendido na Clínica Animal's, localizada no município de Teresina-PI, apresentado sinais compatíveis com hérnia perineal. O diagnóstico definitivo se deu através de exame radiográfico simples, sendo observadas alças intestinais como conteúdo herniário. O tratamento realizado foi a herniorrafia clássica, que se mostrou eficiente. Assim, esse trabalho objetivou relatar um caso de hérnia perineal bilateral em uma gata, descrevendo seus sinais, histórico clinico, método de diagnóstico e tratamento empregado.

Palavras-chave: conteúdo herniário, felino, hérnia bilateral, herniorrafia

\section{Bilateral perineal hérnia in a cat: case report}

Abstract. Perineal hernia is described as the muscle weakening of the perineum region, so that viscera migration to the region may occur. Occurs mainly in dogs, males, uncastrated and elderly. It is uncommon in felines. A female feline, SRD, 7 years old, was seen at the Animal's Clinic, located in Teresina-PI, presenting signs compatible with perineal hernia. The definitive diagnosis was made by simple radiographic examination, and bowel loops were observed as hernial content. The treatment performed was classical herniorrhaphy, which proved to be efficient. Thus, this study aimed to report a case of bilateral perineal hernia in a cat, describing its signs, clinical history, method of diagnosis and treatment employed.

Keywords: hernia content, feline, bilateral hernia, herniorrhaphy

\section{Hernia perineal bilateral en una gata: reporte de un caso}

Resumen. La hernia perineal se describe como el debilitamiento muscular de la región del perineo, de modo que puede ocurrir la migración de las vísceras a la región. Ocurre principalmente en perros, machos, sin castrar y ancianos. Es poco común en felinos. Una hembra felina, criolla, de 7 años de edad, fue atendida en la Clínica Animal, ubicada en Teresina-PI, presentando signos compatibles con hernia perineal. El diagnóstico definitivo se realizó mediante un examen radiográfico simple y se observaron asas intestinales como contenido herniario. El tratamiento realizado fue herniorrafia clásica, que demostró ser eficiente. Por lo tanto, este estudio tuvo como objetivo informar un caso de hernia perineal bilateral en una gata, describiendo sus signos, historia clínica, método de diagnóstico y tratamiento empleado.

Palabras clave: contenido herniario, felino, hernia bilateral, herniorrafía 


\section{Introdução}

Hérnias são descritas como saída de vísceras de sua cavidade natural, por orifícios geralmente formados em locais onde ocorrem fragilidades teciduais (Chevrel \& Rath, 2000). Estas se formam em locais de tecidos mais frágeis como diafragma, região umbilical e períneo que permitem o rompimento de músculos e fáscias ocorrendo assim à passagem de vísceras e consequentemente mudanças relacionadas à sintopia dos órgãos envolvidos (Costa Neto et al., 2006; Fossum, 2014). As hérnias se classificam conforme a região de ocorrência em umbilical, inguinais, diafragmática, perineais e abdominais. Quanto às estruturas envolvidas elas se classificam em hérnias verdadeiras e hérnias falsas. Em relação à presença ou ausência de alteração da função dos órgãos envolvidos elas apresentam as formas redutível e irredutível (Bojrab, 2005; Fossum, 2014; Penaforte Júnior et al., 2017).

A hérnia perineal é resultante da perda da capacidade de sustentação exercida pelo períneo, também chamado de diafragma pélvico (Fossum, 2014). A hérnia perineal ventral é a forma mais comum de hérnia perineal (Bellenger, 1980; Matera et al., 1981), ocorre normalmente pela ruptura de um ou mais músculos que compõem o períneo, formando o anel herniário e permitindo assim a passagem de vísceras que irão se alojar no saco herniário que se forma a partir da ruptura de músculos (Sjollema et al., 1993).

O diagnóstico e feito com base na sintomatologia clínica, considerando suas variações conforme o curso da patologia (Bojrab, 2005), exame físico, exames radiográficos que podem confirmar o envolvimento de órgãos urinários, exames hematológicos e sumário de urina (Bojrab, 2005).

O tratamento da hérnia perineal, uni ou bilateral, consiste em correção cirúrgica. É essencial tratar a sintomatologia e estabilizar o indivíduo antes da realização da intervenção cirúrgica.

\section{Relato de caso}

Felino, fêmea, SRD, de aproximadamente sete anos de idade, com $3,25 \mathrm{~kg}$, foi atendida na Clínica Animal's, em Teresina, no Piauí, quando o tutor do animal relatou que o mesmo apresentava inapetência, aumento de volume na região perineal e tenesmo. Ao exame clínico/físico foi confirmada a presença do aumento de volume em toda extensão da região perineal. Foi solicitado exame radiológico, que contatou a ocupação do saco herniário por alças intestinais contendo fezes em seu interior (Figura 1). O paciente foi então encaminhado para a correção cirúrgica do distúrbio.

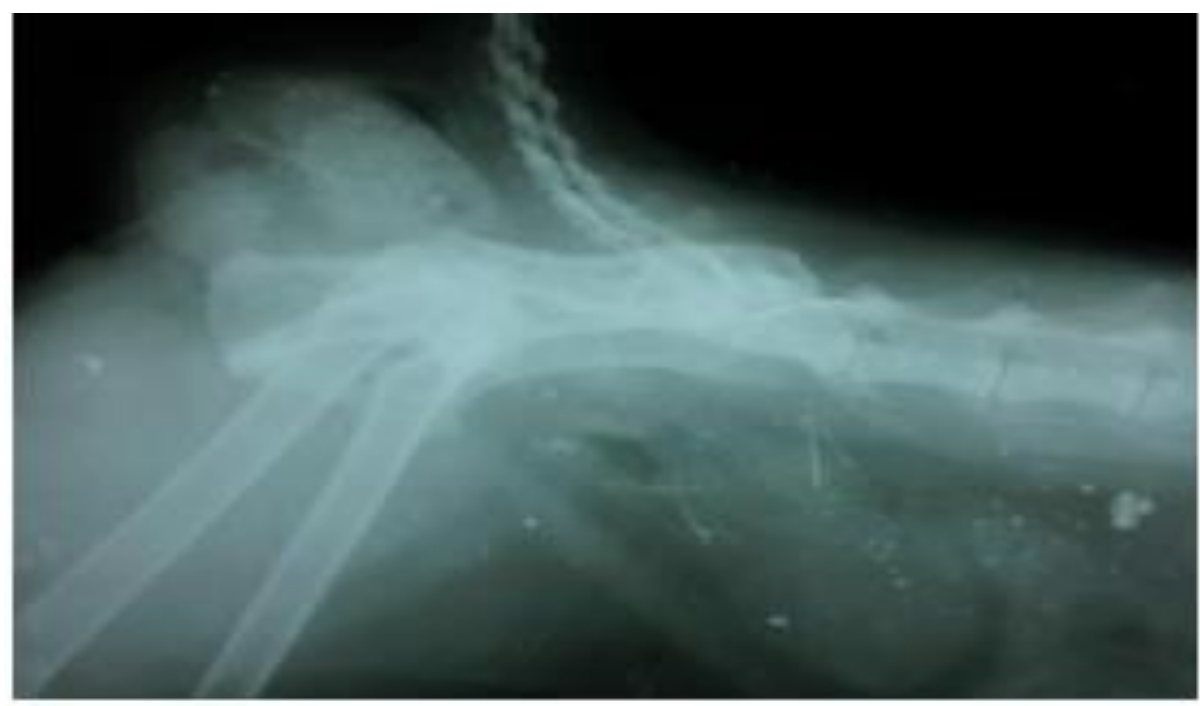

Figura 1. Exame radiográfico demonstrando a presença de alças intestinais como conteúdo herniário.

A correção de seu pela técnica de herniorrafia clássica, que consiste na sutura do esfíncter anal externo aos músculos coccígeo e obturador interno e ao ligamento sacrotuberoso (Figura 3). Com o animal posicionado em decúbito ventral, a cauda fixa por cima do dorso e a região pélvica pouco elevada colocouse uma compressa lubrificada no reto do animal e suturou-se o ânus com uma sutura em bolsa de tabaco, com o intuito de evitar a contaminação fecal do campo cirúrgico (Figura 2). 


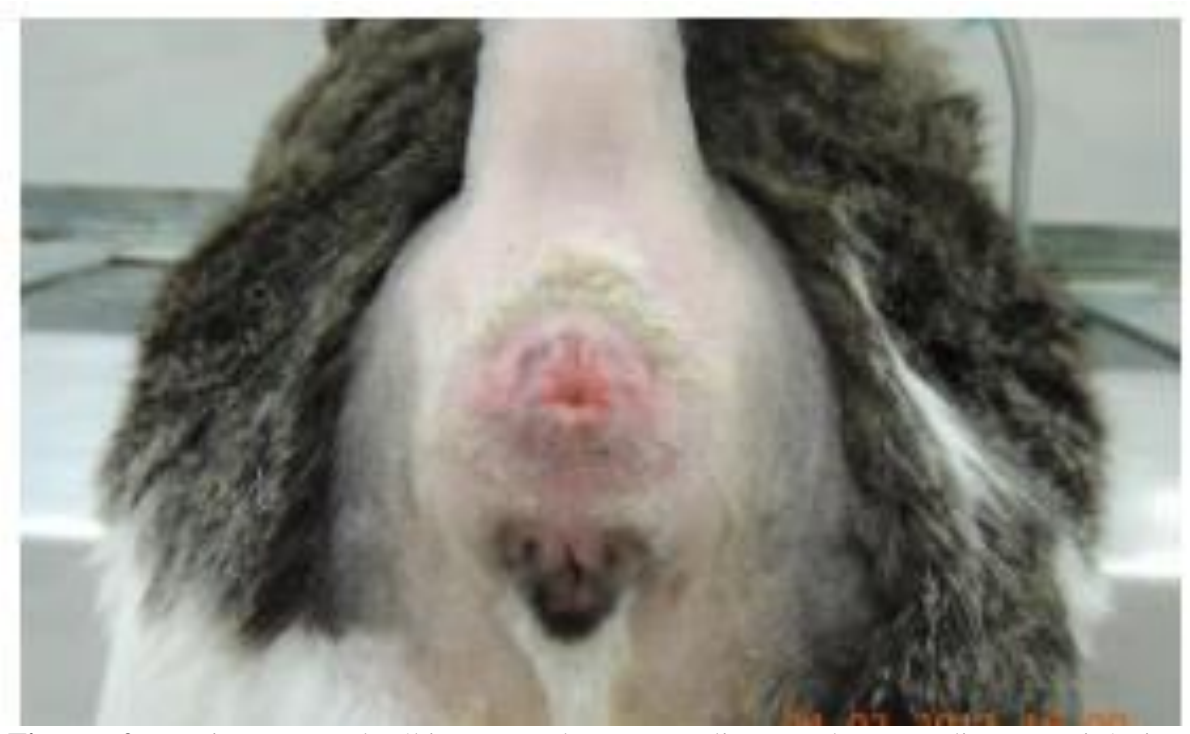

Figura 2. Paciente em decúbito ventral, para realização do procedimento cirúrgico, demonstrando o aumento bilateral da região perineal.

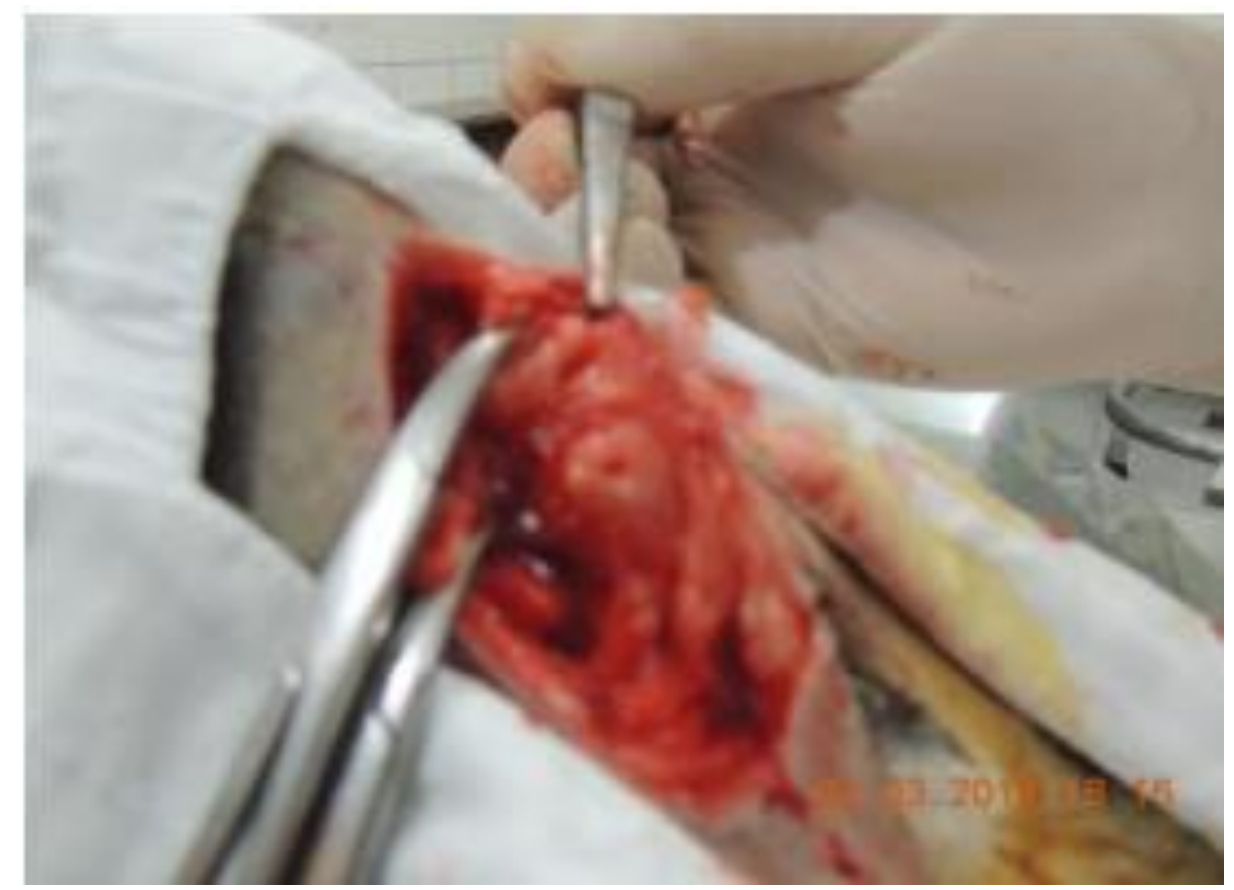

Figura 3. Incisão lateral ao ânus permitindo a sutura dos músculos de sustentação da região perineal

Foram realizadas duas incisões em ambos os lados do ânus, que permitiram a fixação dos músculos e ligamentos que garantem a sustentação da região.

Foi prescrito pelo médico veterinário o fornecimento de alimentação líquida inicialmente, seguida de alimentação pastosa e o retorno gradativo e progressivo a alimentação normal. Foram realizados curativos diários por um período de 10 dias, quando o paciente retornou para retirada dos pontos (Figura 4). O animal foi tratado simultaneamente com antibiótico e anti-inflamatório durante sete e três dias respectivamente.

\section{Resultados e discussões}

No exame radiográfico foi detectada a presença de alças intestinais no interior do saco herniário como descrito por Fossum (2014). Os sinais clínicos apresentados pelo paciente relatado, como tenesmo e aumento da região perineal, coincidem com os sinais descritos por Welches et al. (1992). O animal foi 
acompanhado durante todo o período pós-operatório. O tutor foi fundamental para o sucesso do tratamento instituído ao paciente, como afirmam Acaui et al. (2010).

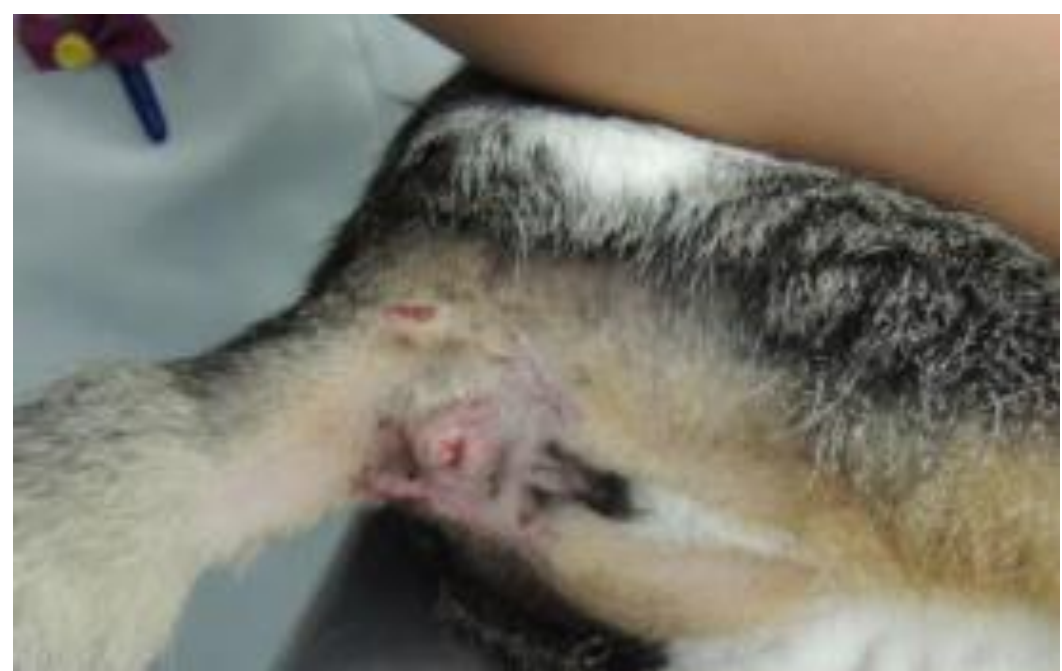

Figura 4. Cicatriz cirúrgica após retirada dos pontos.

\section{Conclusão}

Mediante os resultados obtidos, é conveniente concluir que a herniorrafia clássica foi eficiente no tratamento da hernial perineal bilateral do felino relatado, simultaneamente ao zelo do proprietário em seguir as recomendações do médico veterinário.

\section{Referências bibliográficas}

Acaui, A., Stopiglia, A. J., Matera, J. M., Cortopassi, S. R. G. \& Lacerda, P. M. O. (2010). Avaliação do tratamento da hérnia perineal bilateral no cão por acesso dorsal ao ânus. Brazilian Journal of Veterinary Research and Animal Science, 47(6):439-446.

Bellenger, C. R. (1980). Perineal hernia in dogs. Australian Veterinary Journal, 56(9):434-438.

Bojrab, M. J. (2005). Técnicas atuais em cirurgia de pequenos animais. São Paulo: Editora Roca.

Chevrel, J. P. \& Rath, A. M. (2000). Classification of incisional hernias of the abdominal wall. Hernia, 4(1):7-11.

Costa Neto, J. M., Menezes, V. P., Toríbio, J. M. d. M. L., Oliveira, E. C. S., Anunciação, M. C., Teixeira, R. G. \& Vieira Júnior, A. S. (2006). Tratamento cirúrgico para correção de hérnia perineal em cão com saculação retal coexistente. Revista Brasileira de Saúde de Produção Animal, 7(1):7-19.

Fossum, T. W. (2014). Cirurgia de pequenos animais (4 ed. Vol. 1). São Paulo: Elsevier Brasil.

Matera, A., Moraes Barros, P. S., Stopiglia, A. J. \& Randi, R. E. (1981). Hérnia perineal no cão. Tratamento cirúrgico mediante utilização de malha de polipropileno. Revista da Faculdade de Medicina Veterinária e Zootecnia da Universidade de São Paulo, 18(1):37-41.

Penaforte Júnior, M. A., Aleixo, G. A. S., Maranhão, F. E. C. B. \& Andrade, L. S. S. (2017). Hérnia perineal em cães: revisão de literatura. Medicina Veterinária, 9(1-4):26-35.

Sjollema, B. E., Venker-van, A. H., Hartman, F. \& GoEdegebuure, S. A. (1993). Electromyography of the pelvic diaphragm and anal sphincter in dogs with perineal hernia. American Journal of Veterinary Research, 54(1):185-190.

Welches, C. D., Scavelli, T. D. \& Aronsohn, M. G. (1992). Perineal hernia in the cat: a retrospective study of 40 cases. Journal of American Animal Hospital Association, 28431-438.

Recebido: 18 de setembro, 2019. Aprovado: 2 de dezembro, 2019.

Publicado: 29 de fevereiro, 2020.
Licenciamento: Este artigo é publicado na modalidade Acesso Aberto sob a licença Creative Commons Atribuição 4.0 (CC-BY 4.0), a qual permite uso irrestrito, distribuição, reprodução em qualquer meio, desde que o autor e a fonte sejam devidamente creditados 